\title{
About the Editor
}

\section{Stacy Ho}

Stacy Ho earned her PhD in analytical chemistry in 1997 from the Georgia Institute of Technology (USA). She worked at the Centers for Disease Control and Prevention and for two Contract Research Organizations before joining Genzyme Corporation (MA, USA), a Sanofi company, in 2007. Currently, she is an Associate Director in the Drug Metabolism and Pharmacokinetics Department of the Disposition, Safety and Animal Research organization at Sanofi (MA, USA). Her group provides bioanalytical supports to both discovery and development programs. She has conducted tissue analysis in both discovery and good laboratory practice settings at her current and previous jobs.

For reprint orders, please contact reprints@future-science.com 


\section{Foreword}

\section{Tissue analysis for drug development}

\section{Stacy Ho}

Tissue analysis plays an important role in drug discovery and development. The purpose of tissue analysis varies depending on the type and stage of the drug discovery and development. Initially, tissue analysis is used to verify whether a drug candidate reaches the intended and/or unintended targets. Later, the tissue exposure results provide information on accumulation in tissue after single or repeated doses and on the tissue exposure-dose relationship. The analysis of drugs in multiple tissues from absorption, distribution, metabolism and excretion studies provides the drug tissue distribution information. The drug exposure data in conjunction with biomarker levels in tissue and plasma are used to build pharmacokinetic-pharmacodynamic and/or in silico models, which are used to predict the exposure required for a needed biomarker change and to understand the pathway of efficacy.

Tissue analysis presents many unique bioanalytical challenges, such as the calibration samples not closely mimicking drug binding and distribution in tissue samples obtained from studies, issues of drug extractability and matrix effects. Depending on the species and tissues, analytical considerations and approaches to tissue collection, homogenization, recovery and matrix effects are different. There is no one-size-fit-all solution to those challenges. Operationally wise, tissue analysis is time consuming and resource (labor and material) demanding. Despite these difficulties, tissue analysis has been rapidly gaining momentum in recent years. In my own experience, as recent as 
4-5 years ago, we scarcely saw tissue samples, particularly for drug screening purposes. But in the last 2-3 years, close to half of our studies have tissue samples in addition to plasma. While the use of tissue analysis in my laboratory may not be representative of the greater pharmaceutical industry, from talking to my colleagues, the upward trend is real. This is because the systemic information of drugs such as half-life and volume of distribution at steady state in plasma does not mirror these parameters at the tissue level. Tissue analysis provides valuable and sometimes even critical information to help select and optimize compounds, assessing drug toxicity and efficacy. From the bioanalytical point of view, it is true that tissue analysis results in a disproportionally higher cost and longer analysis time compared with plasma analysis. However, from the overall drug discovery and development point of view, tissue analysis saves time and money because the results help to avoid detours during the drug development process. In fact, tissue analysis is being incorporated throughout the course of drug discovery and development for many programs, such as drugs for oncology and the CNS.

Due to the increased utilization and importance of tissue analysis in drug development, we feel that a book offering a summary of key points on the subject is timely. This compilation describes the typical methodologies and current practices of tissue analysis along with some case studies. It is intended for researchers who have a general knowledge of bioanalysis but may or may not have extensive exposure of tissue analysis in the past. The information in this book is also applicable to tissue samples in other fields, such as environmental monitoring and food testing.

Chapter 1 looks at the current practices and case studies for the analysis of small-molecule drugs in tissue. In this chapter, the authors first present a very useful figure to illustrate the thought process when developing a tissue method. The subsequent discussions and case studies center on the current practices in evaluation of tissue binding, selection of homogenization solvent, determination of amount of solvent used for tissue homogenization, monitoring matrix differences between standards and study sample homogenates, and evaluation of specificity and matrix effects during method development and sample analysis.

Chapter 2 explores tissue homogenization. One of the unique aspects of tissue analysis is that most tissues are solid. To analyze solid tissue, samples have to be homogenized first. It is only right to dedicate an entire chapter to this topic due to both the rich content and the critical role this step plays in successful tissue analysis. Many decisions have to be made to ensure tissues are homogenized properly. The final tissue homogenization methodology is established after carefully considering all of the factors. The chapter discusses 
the types of tissues, tissue homogenization techniques, and the considerations for developing and optimizing tissue homogenization methods.

Chapter 3 discusses the tissue analysis workflow. It describes the key considerations for each step of tissue analysis: proper planning of tissue harvesting and pretreatment, developing a suitable tissue homogenization method, preparing calibration standard and quality control samples using matching or surrogate matrix, and extracting analyte from tissue homogenate. The chapter also outlines the strategies for quantitation of endogenous biomarkers in tissue.

Chapter 4 focuses on method qualification for tissue bioanalysis. Just like analysis of plasma samples, method validity should be established via method qualification or validation prior to analyzing tissue samples. The determination of method precision, accuracy, stability, recovery, matrix effects and homogenate homogeneity are discussed. The chapter also discusses the use of a tiered approach based on the fit-for-purpose principle to balance the desired quality and the level of effort spent. The authors also briefly discuss the regulatory compliance aspects of tissue analysis for data to be included in the submission package.

Chapter 5 considers the practical considerations for the design of tissue pharmacokinetic studies. It discusses the factors affecting tissue distribution, which include physicochemical properties, tissue perfusion and tissue membranes, and transporters. These factors have to be taken in account when selecting tissue, sampling time, dose, dosing regimen, sample collection and assay. In addition, the sampling size and statistical analysis should also be considered when designing tissue pharmacokinetic studies.

Finally, the closing chapter brings together some case studies. Each case study complements the topics discussed in Chapters 1-5, but aims to provide a bigger and more complete picture of the topic. The cases are intended to demonstrate how the tissue analysis is accomplished with the consideration of various factors. They do not cover all scenarios.

In this book, the authors have tried to highlight the critical aspects and considerations of tissue analysis. Due to diversity in tissue analysis, there is no one-size-fit-all solution. The method for analyzing 'my tissue samples' needs to be established based on consideration of relevant factors. Throughout the book, we have tried to summarize the general principles and processes for tissue analysis in order to equip you, the reader, with the knowledge to identify the critical factors and to help you to deal with your particular challenges. We hope that we have accomplished this goal and have provided useful and practical information, which you are able to apply to tissue analysis in your laboratory. 


\section{Acknowledgements}

I would like to express my sincere appreciation and gratitude to all of the authors who took time to write and share their extensive expertise on this topic. It is a demanding process and all authors spent numerous hours on top of their full workload to bring this book to fruition.

\section{Financial \& competing interests disclosure}

The author has no relevant affiliations or financial involvement with any organization or entity with a financial interest in or financial conflict with the subject matter or materials discussed in the manuscript. This includes employment, consultancies, honoraria, stock ownership or options, expert testimony, grants or patents received or pending, or royalties.

No writing assistance was utilized in the production of this manuscript. 
Tissue analysis for drug development 
$\mathrm{HO}$

www.future-science.com 